\title{
Landwirtschaftliche Kolonien deutsch-jüdischer Emigranten in Argentinien
}

\author{
Anne Saint Sauveur-Henn
}

Argentinien war in Lateinamerika dasjenige Land, das zahlenmäßig zwischen 1933 und 1945 die meisten deutschen Emigranten aufnahm - im Verhältnis zur Bevölkerung sogar mehr als die Vereinigten Staaten. Dabei bildete Buenos Aires als Großstadt das Zentrum, da es die meist städtischen Emigranten an Europa erinnerte. Weniger bekannt ist, daß sich eine sehr geringe Minderheit unter den deutsch-jüdischen Emigranten, nach vorsichtigen Schätzungen 4 bis $5 \%$, in landwirtschaftlichen Kolonien niederließ. Selbst wenn es sich nur um etwa 3000 deutsch-jüdische Siedler handelte, scheint eine Untersuchung des originellen Versuchs einer landwirtschaftlichen Kolonisierung im Rahmen einer Emigration aus dem nationalsozialistischen Deutschland von Interesse zu sein.

Zweierlei Quellen liegen hierfür vor: einerseits schriftliche, wie z.B. die Unterlagen der wichtigsten Kolonisationsgesellschaft, ICA genannt, darüber hinaus Berichte im Mitteilungsblatt des Hilfsvereins deutschsprechender Juden in Buenos Aires und vor allem in der Jüdischen Wochenschau, die ab 1940 in Buenos Aires erschien. Andererseits existieren mündliche Quellen, denn die persönlichen Berichte eines ehemaligen ICA-Verwalters, der von 1944 bis 1951 tätig war, sowie von dreizehn deutsch-jüdischen Siedlern, die im Verlauf von drei Forschungsaufenthalten in Argentinien interviewt wurden, erwiesen sich als sehr wertvoll. Nach einem historischen Rückblick sollen die Verteilung der deutsch-jüdischen Emigranten in Argentinien, die Organisation ihrer Rekrutierung, die Niederlassungsbedingungen und die spezifischen Institutionen in den Kolonien dargestellt werden, um dann die Probleme der deutsch-jüdischen Siedler zu schildern und mit einer nuancierten Bilanz zu enden.

\section{Historischer Rückblick}

Um die Immigration deutscher Juden in argentinische Kolonien zu ermöglichen, mußte eine organisatorische Infrastruktur existieren. Nun bestand schon lange eine Organisation, deren ausdrückliches Ziel die Ansiedlung von unterdrückten Juden in eigens für sie geschaffenen Kolonien war, die Jewish Colonization Association, allgemein ICA genannt. Ihr Gründer war Baron Moritz von Hirsch (1831-1896), ein philantropischer reicher Bankier, der am 24. August 1891 in London die ICA mit dem Ziel gründete, "die Auswanderung von Juden aus Europa und Asien zu erleichtern, wo sie durch Ausnahmegesetze unterdrückt und ihrer politischer Rechte beraubt sind, nach anderen Gegenden der Welt, wo sie diese Rechte und alle individuellen Freiheiten genießen können" (Arti- 
kel 3) ${ }^{1}$. Zu diesem Zweck sollten landwirtschaftliche Kolonien in verschiedenen Gegenden Nord- und Südamerikas gegründet werden. Die ICA, die über großes Kapital verfügte, erwarb Ländereien in Brasilien, in den Vereinigten Staaten, in Kanada und später in Israel, vor allem aber in Argentinien, wo sie zwischen 1889 und 1935 über 600.000 Hektar erwarb und siebzehn Kolonien vornehmlich in der Provinz Entre Rios gründete. Die letzten drei Gründungen wurden besonders wichtig für deutsche Juden, insbesondere Avigdor im Jahre $1935^{2}$.

Zwei weitere private Organisationen neben der ICA sind zu nenne: Die eine war eine allgemein jüdische, die "Sociedad de Protección a los inmigrantes israelitas", SOPROTIMIS genannt, die mit der ICA zusammenarbeitete, und eine rein deutsch-jüdische, der am 26.4.1933 gegründete "Hilfsverein deutschsprechender Juden", der etwa 350 Kolonisten unterstützte, darunter Lehrlinge im eigenen Umschulungszentrum. ${ }^{3}$

Eine Ansiedlung deutscher Juden in landwirtschaftlichen Kolonien erschien als ein Ausweg, um - wie die Archive der ICA zeigen - gesetzliche Restriktionen zu umgehen. Selbst wenn die ICA wegen der wirtschaftlichen Schwierigkeiten zögerte, eine neue Siedlung zu gründen, erkannte sie 1933, daß auf diese Weise leichter Visen erlangt werden konnten, und entschloß sich im März 1935, für die deutschen Juden eine eigene Kolonie in Avigdor zu gründen. Nach dem argentinischen Dekret vom 28. Juli 1938, das durch die Forderung nach zusätzlichen Dokumenten die Einwanderung erschwerte, protestierte die ICA gegen die neuen Restriktionen und erreichte dadurch die Einreiseerlaubnis für die von ihr unterstützten Siedler. Im September 1939 wurde es den Konsuln erlaubt, Juden, die keine Bauern waren, Einreiseerlaubnisse zu erteilen, wenn sie von der ICA empfohlen worden waren, und die ICA verpflichtete sich im Gegenzug, jeden Siedler anzuzeigen, der innerhalb von zwei Jahren seinen Hor verlassen würde. Doch der Krieg unterbrach diese Möglichkeit, und obwohl in verschiedenen Kolonien noch Platz für Siedlerfamilien gewesen wäre, kamen aufgrund der politischen Situation in Deutschland ab 1940 keine weiteren jüdischen Siedler mehr. ${ }^{4}$

${ }^{1}$ Simon Weil: Las colonias de la ICA. In: 50 años de colonización judia en la Argentina. 1939. Buenos Aires: DAIA, 148.

${ }^{2} J$ wewish Colonization Association (ICA). 1941. Jewish Colonization Association: Su obra en la república Argentina: 1891-1941. Buenos Aires: Ayacucho, 14f. et passim.

${ }^{3}$ Sociedad de protección a los inmigrantes israelitas. 1943. Bericht der SOPROTIMIS. In: Zehn Jahre Aufbauarbeit in Südamerika, $\mathrm{Hg}$. anläßlich des zehnjährigen Bestehens der Asociación Filantrópica Israelita, Buenos Aires, 70-77; Zehn Jahre sozialer Arbeit, ebda., 22-36.

${ }^{4}$ Avni 1983, 404f., 417, 433-436, 445ff. 


\section{Die Verteilung der deutsch-jüdischen Siedler}

Insgesamt konnte die ICA über 3000 deutsch-jüdische Siedler in Argentinien unterbringen. Die Kolonisationsgesellschaft wandte dabei zweierlei Methoden an: Sie gründete einerseits 1935 für deutsch-jüdische Emigranten eine eigene Kolonie, Avigdor, und integrierte andererseits, insbesondere in den Jahren 1937 und 1938, deutsche Juden in bereits bestehende Kolonien. Die Ansiedlung erfolgte in drei Provinzen. In der Provinz Entre Rios 700 Kilometer nördlich von Buenos Aires wurden etwa 166 Familien angesiedelt: etwa 120 Familien in Avigdor, einer rein deutsch-jüdischen Kolonie von über 17000 Hektar, in der die ersten deutsch-jüdischen Emigranten im Januar 1936 und die letzten 1940 ankamen. Etwa 50 Familien kamen 1938 und 1939 in die nahe bei Avigdor gelegenen Kolonien Louis Oungre und Leonhard Cohen (auch Alcaraz genannt), die 1925 bzw. 1932 gegründet worden waren und in denen bereits Söhne von früher eingewanderten russischen Siedlern und polnische Juden lebten. In der Provinz Santa Fé wurden 80 Familien in der Colonie Moiseville angesiedelt. Moiseville, 1891 gegründet, war die größte und die älteste, auch die wohlhabendste der ICA-Kolonien, in der ursprünglich russische Juden lebten und 1938 tschechische und deutsche Juden hinzukamen. In der Provinz Buenos Aires bzw. Pampa wurden 20 Familien in der Colonie Baron Hirsch, auch Rivera genannt, etwa $550 \mathrm{~km}$ südlich von Buenos Aires, angesiedelt. In Rivera, 1904 gegründet, lebten bereits russische und polnische Juden. ${ }^{5}$

\section{Die Rekrutierung der Siedler}

Die Rekrutierung der Juden in Deutschland für diese Kolonien war mit Problemen verbunden. Die ICA koordinierte die Arbeit in Argentinien und in Deutschland. Funktionäre der ICA reisten durch Deutschland, um mit Familien Kontakt aufzunehmen, die in eine südamerikanische Kolonie emigrieren wollten. Das Pariser Büro spielte dabei eine große Rolle. So erzählten zwei ehemalige Kolonisten aus Avigdor, daß sie in Paris ein Visum der ICA bekommen hätten. ${ }^{6}$ Laut einem ehemaligen ICA-Verwalter suchte die ICA vor allem in Hessen, in der Frankfurter Gegend, in Osteuropa und in Berlin Siedler zu rekrutieren. Ursprünglich suchte die ICA Emigrationskandidaten, die Erfahrung in der Landwirtschaft hatten, doch stieß sie auf große Schwierigkeiten aufgrund der Berufsstruktur der deutschen Juden, von denen nur eine kleine Minderheit Landwirte waren, die infolgedessen nicht umgeschult werden mußten. ${ }^{7}$ Die ICA suchte Lösungen für dieses Umschulungsproblem. Einerseits schuf sie in

${ }^{5}$ Kolonisierung jüdischer Familien aus Deutschland durch Jewish Colonization Association. 1943. In: Zehn Jahre Aufbauarbeit in Südamerika, 114. - Simon Weil: Las colonias de la ICA. In: 50 años de colonización judia en la Argentina. 1939, 156f., 160-165, 188-190.

${ }^{6}$ Interviews Herr und Frau Kahn, Herr und Frau S., Buenos Aires, 15.4.1978.

${ }^{7}$ Interview Roberto Schopflocher, Buenos Aires, 29.9.1990. 
Deutschland Ausbildungszentren, wie z.B. in Neuendorf, doch wurden nur wenige auf diese Weise ausgebildet, und selbst nach einer solchen Umschulung waren nach Aussage eines Kolonisten aus Rivera die zukünftigen Siedler nicht ausreichend auf ihre neue Arbeit in Argentinien vorbereitet. ${ }^{8}$

Andererseits schuf die ICA ein originelles Rekrutierungsprinzip. Nach den Dokumenten der ICA zu urteilen, die sie anläßlich ihres 50jährigen Bestehens veröffentlichte, wurden anfänglich von 1933 bis 1936 nur Landwirte rekrutiert, dabei jedoch das Prinzip des "Vorfahrers" eingeführt: Ein Mitglied der Familie (der Vater oder der älteste Sohn) mußte zuerst für mindestens drei bis sechs Monate allein nach Argentinien fahren, um bei einem in Argentinien schon niedergelassenen Kolonisten die im Lande betriebene Landwirtschaft und die Sprache zu erlernen; dann erst durfte die übrige Familie nachkommen. So wurden 1937 und 1938175 "Vorfahrer" ausgebildet, einige in einem Lehrbetrieb in Avigdor, die anderen bei Kolonisten. Der "Vorfahrer" wurde jeden Monat geprüft, und wenn er genügend vorbereitet schien, durfte seine Familie nachkommen. ${ }^{9}$ Mit der Zeit jedoch wurde auf dieses Prinzip verzichtet. Nach Angabe eines Kolonisten aus Avigdor spielte die gespannte Situation in Deutschland eine große Rolle bei diesem neuen Pragmatismus; nach Unterlagen der ICA war auch das argentinische Dekret vom 28.7.1938 entscheidend, wonach nur Familien als Einwanderungskandidaten angenommen werden konnten. ${ }^{10}$

Das Prinzip des "Vorfahrers" brachte große persönliche Probleme mit sich, von denen die Berichte zweier ehemaliger Siedler aus Avigdor Zeugnis geben. Die Familie einer Frau, die als Baby in Avigdor ankam, stammte aus Worms. Ihr Vater und ihr Onkel erfuhren von der ICA, daß einer der beiden Brüder als erster fahren mußte. Da der eine drei kleine Kinder hatte und der andere vier, wollte keiner gerne allein fahren. So losten sie, der Vater mußte gehen, und die Mutter erzählte oft, wie schwer ihr dieses Jahr unter schwierigen Umständen gefallen war. Ein damals 17 jähriger Junge wollte ursprünglich nach Palästina emigrieren, doch der Vater erfuhr von der ICA, daß die Familie nach Argentinien fahren dürfte, vorausgesetzt, daß einer vorführe. So verzichtete der Junge auf sein ursprüngliches Vorhaben und fuhr im April 1937 schweren Herzens allein nach Argentinien. ${ }^{11}$

Dank dem Zeugnis zweier Kolonisten aus Avigdor konnte noch ein anderes, in keinen offiziellen Unterlagen dargestelltes Prinzip entdeckt werden, das des "Beifahrers". Damit mehr Juden aus Deutschland emigrieren konnten, nahm eine Familie noch einen Jungen oder ein junges Paar als Familienmitglieder mit. So nahm der Vater eines Kolonisten, der als Elfjähriger 1938 in Avigdor

\footnotetext{
${ }^{8}$ Interview Alfred Lewkowitz, Buenos Aires, 20.9.1990.

${ }^{9}$ Jewish Colonization Asociation: Su obra en la república Argentina, $18 \mathrm{f}$.

${ }^{10}$ Ebda., 33; Interview Pablo Herrn May, Buenos Aires, 1.10.1990.

${ }^{11}$ Interviews Frau Lichtenstein, Buenos Aires, 4.10.1990 und Roberto May, Buenos
} Aires, 1.10.1990. 
ankam, ein Paar und zwei Jungen mit. Einer der beiden war dafür eigentlich nicht vorgesehen gewesen, doch der Vater hatte erzählt, daß der Junge von den Nazis verfolgt wurde und dies der einzige Ausweg sei, ihn zu retten. So kam er mit, und die ganze Gruppe betrachtete sich von nun an als eine einzige Familie. Sie verfügten nur über ein Haus mit zwei Zimmern und einer Küche. Erst viel später trennten sich die "Beifahrer" von der Familie, als sie erkannten, $\mathrm{daß}$ es für sie in Avigdor keine Zukunft gab. ${ }^{12}$

\section{Die Niederlassungsbedingungen}

Einmal von der ICA rekrutiert, unterlagen alle Kolonisten denselben Niederlassungsbedingungen. Die ICA lieferte ein Stück Land von etwa 75 Hektar, ein Haus, einen Hühnerstall, eine Scheune, Vieh, landwirtschaftliche Geräte. Manchmal übernahm der Kolonist die Unkosten (15.000 pesos), aber meistens lieh die ICA die nötigen Gelder. Der Siedler schloß mit der ICA einen Pachtvertrag für ein Jahr ab, der automatisch für fünf Jahre verlängert wurde, und konnte nach acht Jahren Eigentümer werden, wenn er das Inventar und die Zinsen auf den Preis des Landes zurückbezahlt hatte; den Rest des Betrages konnte er in neun Jahren bezahlen. ${ }^{13}$ Auch bestand eine Wiederverkaufsklausel, wonach die ICA innerhalb von drei Jahren das Land für denselben Preis wiedererlangen konnte. Um 1955 mußte die ICA aufgrund veränderter Steuergesetze, durch die sie zu sehr benachteiligt worden wären, sämtliche Länder an die übriggebliebenen Siedler verkaufen. ${ }^{14}$ In Avigdor waren alle Häuser in Blocks von zwei bis vier Kolonistenfamilien angeordnet, die sich eine gemeinsame Pumpe teilten; in den älteren Kolonien bestand zum Teil noch die ursprüngliche Siedlungsmethode der ICA mit der Zusammenfassung aller Häuser in einem Zentrum. ${ }^{15}$

\section{Die spezifischen Institutionen}

In diesen jüdischen Kolonien enstanden spezifische Institutionen, wie sie das Beispiel der rein deutsch-jüdischen Kolonie von Avigdor am besten zeigt. Die wichtigste Institution war in jeder Kolonie die landwirtschaftliche Kooperative. Die erste Kooperative in Argentinien überhaupt wurde 1900 in einer jüdischen Kolonie (Basavilbaso) gegründet. In Avigdor wurde sie gleich anläßlich der Gründung 1936 mit Unterstützung der ICA geschaffen und funktionierte als Konsum- und Produktionsgenossenschaft. Die ICA übernahm ebenfalls die Gründung einer Schule, die sie der argentinischen Regierung zur Verfügung stellte. In Avigdor wurde die kleine, einklassige argentinische Schule von einer spezifisch jüdischen Ausbildung zweimal wöchentlich ergänzt.

\footnotetext{
${ }^{12}$ Interview Curt Lichtenstein, Buenos Aires, 4.10.1990.

${ }^{13} 50$ Jahre ICA Arbeit in Argentinien (1891-1941). In: Filantrópica IX (100), Juli 1942: 29.

${ }^{14}$ Interview Roberto Schopflocher.

${ }^{15}$ Jewish Colonization Association: Su obra en la república Argentina, 37.
} 
Diese Kolonien wollten ausdrücklich jüdische Kolonien sein. So steht in einem Artikel der Jüdischen Wochenschau vom 21.8.1942 über Avigdor:

Für diese Kolonisten gibt es nur eine Lösung: die jüdische. Sie haben nicht alte Bande, die sie aufrechterhalten, sie haben keine Verbindung mehr mit jenen, die zu ihren Henkern gehören [...] Sie wollen sich mit dem Boden ihrer neuen Heimat verbinden, sie werden sich mit Argentinien verwurzeln [...] aber sie sind Juden, sie bleiben Juden und wollen es auch sein. Als Juden siedeln sie, als Juden leben sie, als Juden und als bewußte, mit ihrem Volk verbundene Juden, wollen sie sterben. ${ }^{16}$

So spielte die Synagoge in den Kolonien eine entscheidende Rolle. In Avigdor bestanden auf Grund der Entfernung der Kolonisten vom Zentrum vier Gebetszentren, und in jedem Bericht der Jüdischen Wochenschau über die Einweihung einer neuen Synagoge merkt man, daß die Synagoge eine zentrale Stellung im Judentum innehatte, als Erinnerung an das vernichtete Gotteshaus in der Heimat, als Sieg des Geistes über die Zerstörung. ${ }^{17}$

Vor allem das intensive kulturelle Leben unterschied Avigdor von anderen Kolonien. Urheber desselben war Dr. Alfred Neumeyer, ehemaliger höherer Richter in Bayern und Präsident der jüdischen Gemeinde von München sowie Mitbegründer der "Reichsvertretung der deutschen Juden". Dieser alte Herr versammelte jeden Freitag die Kolonisten, hielt Vorträge über Platon und Goethe und schuf ein Kulturzentrum mit einer großen Bibliothek. In den Erzählungen von Augenzeugen ist spürbar, wie auffallend der Kontrast war zwischen dieser beeindruckenden Bibliothek mit von den Emigranten 1936-1937 mitgebrachten Büchern und dem äußerst primitiven Haus mit dem gestampften Boden und der Petroleumlampe - ein Charakteristikum der deutsch-jüdischen Kolonisation überhaupt. ${ }^{18}$ Im Kulturzentrum, Salon genannt, fanden Konzerte und Theateraufführungen statt, von denen einige auch in Buenos Aires gezeigt wurden. ${ }^{19}$ Dies hängt vielleicht mit der spezifisch rein deutsch-jüdischen Rekrutierung in Avigdor zusammen: Nach Aussage eines Kolonisten blieben die deutschen Juden unter sich, sie fühlten sich als Jecke; nach einem anderen Kolonisten sprachen die in Avigdor arbeitenden Argentinier zum Teil besser deutsch als die deutschen Kolonisten spanisch. Doch wurde, speziell bei der Jugend, großer Wert auf die Erlernung der spanischen Sprache gelegt. ${ }^{20}$ In anderen Kolonien, in denen die deutschen Juden in der Minderheit waren, unterhielten sie sich mit den Ostjuden zunächst auf Jiddisch. In Rivera siedelten

\footnotetext{
${ }^{16}$ Avigdor. In: Jüdische Wochenschau III (122), 21.8.1942.

${ }^{17}$ S. z.B.: Aus der Arbeit der ICA. In: Jüdische Wochenschau I (1), 26.4.1940: 9.

${ }^{18}$ Interview Roberto Schopflocher.

${ }^{19}$ Einweihung eines Kulturheimes in der Colonia Avigdor. In: Jüdische Wochenschau II (47), 14.3.1941: 12 .
}

${ }^{20}$ Neumeyer, Alfred. 1943. Avigdor. In: Zehn Jahre Aufbauarbeit in Südamerika, 160. 
die deutschen Juden zum Teil zusammen und bildeten die "Gruppe Starkmeth" mit eigenen Institutionen. ${ }^{21}$

\section{Die Schwierigkeiten der deutsch-jüdischen Kolonisten}

Selbstverständlich stießen die deutsch-jüdischen Siedler auf vielerlei Probleme materieller, landwirtschaftlicher, beruflicher und persönlicher Art, von denen sowohl die Presseberichte ein Bild geben als auch und vor allem die persönlichen Zeugnisse der Kolonisten. Das erste materielle Problem, mit dem die Siedler konfrontiert wurden, war die Reise. In Buenos Aires angekommen, mußten sie meist in den überfüllten "Einwandererhotels" übernachten, anschlieBend zwei Tage lang 700 Kilometer mit Zug, Fähre und Lastwagen fahren. Diese Isoliertheit machte den meist städtischen Kolonisten zu schaffen, zumal die Wege nicht geteert waren und es in Avigdor bei Regen sechs bis acht Stunden statt einer Stunde bei normalem Wetter erforderte, um das Zentrum zu erreichen, sowie zwei bis drei Tage bis zur Bahnstation Bovril, die 25 Kilometer entfernt war. ${ }^{22}$

Die Wohnbedingungen bedeuteten für die Kolonisten eine zusätzliche Schwierigkeit. Bei der Ankunft in Avigdor sagte die Mutter eines Kolonisten: "Es ist schlimmer als ein Stall, bei uns wohnen die Kühe besser". ${ }^{23}$ Nach Aussage aller interviewten Kolonisten waren die Häuser recht primitiv, ohne Strom und Wasser; zwei bis vier Familien mußten sich eine Pumpe teilen. Der Unterschied zur Heimat war hierin besonders kraß. Herr May aus Avigdor hob hervor, daß es Strom erst 1971 gab, in seinem Heimatdorf in Deutschland aber schon 1906.

Den spät angekommenen Emigranten, vor allem denen, die 1939 und 1940 in die landwirtschaftlichen Kolonien kamen, fehlte es oft an materiellen Gütern. So schrieben z.B. 1940 und 1941 einige Kolonisten aus Rivera und Avigdor verzweifelte Briefe an die Jüdische Wochenschau: es fehle nicht nur an Geld, sondern auch an Kleidern, Betten, Möbeln, Decken usw. ${ }^{24}$ Die früher eingewanderten Kolonisten konnten dagegen ihre Güter mitnehmen, so daß einem Rabbiner aus Buenos Aires, der 1942 Avigdor besuchte, der krasse Gegensatz zwischen "der Einrichtung aus der guten alten Zeit in Deutschland" und "dem sonstigen Lebensstandard" auffiel. ${ }^{25}$ Diese Probleme rührten von der "Verpflanzung" der Emigranten her - wie es Herr Schopflocher formulierte: "Man

\footnotetext{
${ }^{21}$ Bericht aus der ICA-Kolonie Baron Hirsch. In: Jüdische Wochenschau I (8), 6.1940: 3.

${ }^{22}$ Interviews Pablo May, Buenos Aires, 1.10.1990, Herr und Frau Harf, Herr und Frau Marx, Buenos Aires, 9.11.1977.

${ }^{23}$ Interview Frau Lichtenstein.

${ }^{24}$ Bericht aus der ICA-Kolonie Baron Hirsch. In: Jüdische Wochenschau I (8), 6.1940:

3; ICA-Siedler antworten. In: Jüdische Wochenschau II (42), 7.2.1941: 7

${ }^{25}$ Günter Friedländer: Bei den Kolonisten in Avigdor.In: Jüdische Wochenschau III (98), 6.3.1942: 8 .
} 
hat Leute dorthin verfrachtet, die aus einer anderen Zivilisation kamen". Oder wie es Herr May aus Avigdor ausdrückte: "Eine verlassene Gegend ohne Strom, ohne Wege, mit Entbehrungen, das war nicht jedermanns Sache".

Auch rein landwirtschaftliche Probleme machten den deutsch-jüdischen Kolonisten zu schaffen. Die Einnahmen waren nicht stabil, sie hingen sehr von den Witterungsverhältnissen und der wechselnden Nachfrage ab. Die Ernten waren oft durch Seuchen gefährdet. Am schlimmsten waren nach allen Zeugnissen die Heuschrecken, die ganze Felder innerhalb von fünf Minuten vernichten konnten. Nach den Briefen von Kolonisten an die Jüdische Wochenschau hatten einige von ihnen große, manchmal auch dramatische finanzielle Probleme. ${ }^{26}$

Eine beträchtliche Schwierigkeit bestand sicherlich in der mangelnden beruflichen Eignung und Ausbildung der Siedler, denn die meisten Emigranten hatten gar keine Erfahrung in der Landwirtschaft. Alle Gesprächspartner betonten dieses wichtige Charakteristikum: $95 \%$ der deutsch-jüdischen Kolonisten waren keine Landwirte; im besten Fall waren sie Gärtner oder Viehhändler, die meisten aber waren zuvor Kaufleute und kamen fast ohne jede Vorbereitung nach Argentinien. Siedler aus Großstädten wie Berlin und München hatten die größten Anpassungsprobleme. Manche hatten nach eigenen Aussagen noch nie eine Kuh gesehen. Viele Anekdoten werden überliefert, in denen die oft völlige Ahnungslosigkeit der Immigranten in dieser Hinsicht angesprochen wird. Doch selbst für die wenigen, die in Deutschland in der Landwirtschaft gearbeitet hatten, war die Umstellung wegen der ganz anders gearteten landwirtschaftlichen Bedingungen sehr schwer. Zwar wußte man von Anfang an um diese Probleme, doch, wie es Dr. Kurt Riegner formulierte, der von 1942 bis 1950 Lehrer in Avigdor war: "Um Menschen zur retten, war jeder Kniff möglich". ${ }^{27}$

Um diese Schwierigkeiten zu bewältigen und eine landwirtschaftliche Umschulung zu sichern, wurde nicht nur von der ICA, sondern auch vom Hilfsverein deutschsprechender Juden in Buenos Aires 1936 eine Lehrfarm in ChoeleChoel in der Provinz Rio Negro im Süden Argentiniens gegründet, getragen von dem "Fomento Agrícola Adolfo Hirsch", der ein Landgut von vierzig Hektar erwarb. Ursprünglich hatte der Hilfsverein große Pläne: Er wollte dieses öde Gelände urbar machen und dabei jugendliche Emigranten von siebzehn bis zweiundzwanzig Jahren in der Landwirtschaft, besonders im Obstbau, ausbilden, dann kleine Höfe gründen und diese an die Kolonisten verkaufen. ${ }^{28}$

Doch die Wirklichkeit sah anders aus, wie es ein Siedler schilderte, der als einer der ersten Lehrlinge 1936 mit 17 Jahren in Choele-Choel ankam und bis zum Ende dort blieb. Zwar schrieb Herr Hirschmann 1943 anläßlich des zehn-

\footnotetext{
${ }^{26}$ ICA-Siedler antworten. In: Jüdische Wochenschau II (39), 17.1.1941: 10; Jüdische Wochenschau II (42), 7.2.1941: 7 .

${ }^{27}$ Interview Dr. Kurt Riegner, Buenos Aires, 9.1.1992.

${ }^{28}$ Mitteilungsblatt des Hilfsvereins deutschsprechender Juden IV (46), 12.1937: 5-8.
} 
jährigen Bestehens des Hilfsvereins positiv über die im Umschulungszentrum geleistete Arbeit, in dem die "neuen Landwirte" heiter seien und sich in Argentinien sicher fühlten ${ }^{29}$, doch nach mehr als fünfzig Jahren war die mündlich gegebene Bilanz nuancierter. Das doppelte Projekt einer Umschulung und einer Kolonisierung sei für ihn ein "totgeborenes Kind" gewesen. Zwar sei das Hauptziel erreicht worden, daß etwa 150 junge deutsche Juden Deutschland verlassen konnten, doch hätten die wenigsten von der Ausbildung profitiert und seien zu $99 \%$ nach Buenos Aires zurückgekehrt. Darunter bildeten die, die als Gärtner gearbeitet hätten, eine Minderheit; die meisten kehrten zu kaufmännischen Berufen zurück. Der Mißerfolg war dieser Schilderung nach auf verschiedene Gründe zurückzuführen. Die Rekrutierung der jungen Leute sei keiner Selektion unterworfen worden, es hätten sich darunter auch richtige Proleten und Trinker befunden, verlorene Burschen. Viele seien aus der Stadt gekommen. Außerdem hätten die Lehrlinge dort zwar gearbeitet, doch nicht richtig gelernt, zumal der Ausbilder kein guter Pädagoge gewesen sei. Als 1940 das Gelände verkauft wurde, blieben nur zwei Familien, darunter unser Gesprächspartner. ${ }^{30}$

Bedingt durch den beruflichen Ursprung der deutsch-jüdischen Emigranten entstanden oft auch persönliche Probleme. Die alten Leute litten am meisten unter der totalen Entwurzelung. Nach Aussage von Herrn May aus Avigdor konnten die alten Leute in den landwirtschaftlichen Kolonien die Emigration nie überwinden; sie blieben im Geiste in Deutschland, lernten nie Spanisch und konnten sich nicht umstellen. Aber auch für die jungen Leute war die Umstellung mühsam. So berichteten Kolonisten aus Rivera 1939 im Mitteilungsblatt des "Hilfsvereins deutschsprechender Juden", wie schwer sich die Jugend an die neue Atmosphäre anpassen könne, wie groß der Unterschied sei zu ihrer Zeit in Deutschland, wo sie ein gesellschaftliches Leben und intellektuelle Zerstreuung gehabt hätten. Jetzt müßten sie hart arbeiten, hätten Sorgen um das tägliche Brot und litten stark unter dem Eindruck, von der gebildeten Welt abgeschnitten und an die Eltern gebunden zu sein. ${ }^{31}$ Ein soziologischer Faktor verstärkte noch die Probleme der Jugend: In den Kolonien fehlte es an Mädchen. Viele Familien schickten nämlich ihre Töchter nach Buenos Aires, wo sie in einer Familie arbeiteten und den Eltern in der Kolonie Geld schicken konnten. Ein Kolonist berichtete, wie die ICA sogar fünfzehn Mädchen aus einem Waisenhaus von Buenos Aires nach Avigdor schickte, und tatsächlich heirateten einige. ${ }^{32}$

${ }^{29} \mathrm{Hirschmann/Leimdorfer:} \mathrm{Fomento} \mathrm{Agrícola} \mathrm{Choele-Choel.} \mathrm{In:} \mathrm{Zehn} \mathrm{Jahre} \mathrm{Aufbauarbeit}$ in Südamerika. 1943, 166.

${ }^{30}$ Interview Hermann Hirschmann, Buenos Aires, 25.9.1990.

${ }^{31}$ Einwanderung. In: Mitteilungsblatt des Hilfvereins deutschsprechender Juden. VI (63), 6.1939: 6 .

${ }^{32}$ Interview Pablo May. 
Manchmal waren die Beziehungen der Kolonisten untereinander nicht einfach. Nach dem Zeugnis zweier ICA-Verwalter aus Avigdor war das Hauptproblem die für zwei bis vier Kolonisten gemeinsame Pumpe, die ursprünglich von der ICA als Mittel gegen die Isolation gedacht wurde, an der aber immer wieder Streitigkeiten entstanden. ${ }^{33}$ Auch mit den ICA-Verwaltern entstanden manchmal Probleme. Mehrere Avigdor-Kolonisten schilderten den damaligen Verwalter als sehr autoritär ("ein Diktator"). Er habe eine preußische Disziplin einführen wollen und starken Druck auf die Kolonisten ausgeübt, damit sie die Kolonie nicht verließen.

\section{Bilanz}

Will man eine Bilanz der deutsch-jüdischen Kolonisation in Argentinien ziehen, so wird sie notwendigerweise nuanciert sein. Doch muß mit einer wesentlichen Feststellung begonnen werden: Wie es bei allen mündlichen und schriftlichen Zeugnissen auffiel, wird keine Kritik die tiefe Dankbarkeit der Kolonisten verwischen können, die dem Tod entrinnen und in Argentinien eine neue Existenz aufbauen konnten. Ein Kolonist aus Avigdor formulierte es so: "Man war froh, seine Haut gerettet zu haben". Tatsächlich betrachteten einige Kolonisten trotz der extremen Schwierigkeiten zu Beginn der Ansiedlung die Kolonie als ihre zweite Heimat. ${ }^{34}$ Der andere unbezweifelbare Erfolg lag auf landwirtschaftlicher Ebene: Dank der Arbeit der Kolonisten genoß Avigdor z.B. schon 1943 ein hohes Ansehen bei argentinischen Gouverneuren und Ministern, die die Kolonie besuchten. Nach Aussage von Herrn Schopflocher beeinflußten die ICA-Kolonien das landwirtschaftliche Leben in Argentinien in zweierlei Hinsicht: durch ihre Organisation in Kooperativen einerseits, sowie durch die Einführung einer Mischwirtschaft mit Hühnerzucht und Milchproduktion. Avigdor galt als Musterkolonie und als Beweis des Erfolgs dank des Lebenswillens der deutsch-jüdischen Emigranten.

Andererseits tauchten zwei Probleme auf: die Fehler der ICA und die Abwanderung. Nach dem Urteil eines ehemaligen ICA-Verwalters, Herrn Schopflocher, beging die ICA einige Fehler. Die Zentren in Paris und London seien viel zu bürokratisch gewesen, die Verwaltung "verknöchert". Erst 1950 habe im Verwaltungsrat der ICA auch ein Vertreter der argentinischen Juden gesessen. Wäre die ICA-Verwaltung nicht so starr gewesen, hätten sicherlich mehr deutsche Juden gerettet werden können. Gewiß war die ICA nicht verantwortlich für die Berufsstruktur der deutschen Juden, doch waren die Beziehungen zwischen ihr und den Kolonisten oft zu paternalistisch. Die Wahl der Ausbilder wurde nicht immer effizient gehandhabt: Die entsprechende Person in Avigdor war Österreicher und sprach kaum Spanisch. Außerdem waren die

${ }^{33}$ Bei den Kolonisten in Avigdor. In: Jüdische Wochenschau III (98), 6.3.1942: 8.

${ }^{34}$ S. z.B. Bericht der Familie W. an Günter Friedländer, In: Jüdische Wochenschau III (98), 6.3.1942: 8 . 
Ausgangsbedingungen schlecht, die Kolonie mitten im Urwald zu isoliert und die Höfe mit 75 Hektar zu klein. ${ }^{35}$

Das zweite Problem war das der Landflucht. Von Anfang an verließen einige Siedler die Kolonie, um in die Stadt zu ziehen. Herr May aus Avigdor berichtete von einem besonders kurzen Aufenthalt: 1940 habe er eine Familie, die mit zwei Kindern ankam und deren Ausbilder er werden sollte, zu ihrem Hof begleitet. Am nächsten Morgen jedoch stellte er fest, daß sie in der ersten Nacht die zehn Kilometer bis zum Zentrum gelaufen und wieder abgereist war. Familien, die es sich finanziell erlauben konnten, gingen sogleich wieder. Schon 1943 betrachtete Dr. Neumeyer die Abwanderung in die Großstadt als eines der wesentlichen Probleme der Kolonie, zumal wenn junge Leute die Kolonie verließen. ${ }^{36}$

Wenn diese Abwanderung also schon vor dem 2. Weltkrieg einsetzte, so brach sie in vollem Ausmaß nach diesem aus. In Avigdor verkauften die deutsch-jüdischen Kolonisten ihre Ländereien nicht immer an jüdische Siedler, denn sie konnten - sobald sie schuldenfrei geworden waren - frei über ihr Land verfügen. Heute sind etwa zehn deutsch-jüdische Familien übriggeblieben, die zum Teil in Bovril (der Bahnstation) wohnen, wo sie also eigentlich keine Kolonisation mehr betreiben. ${ }^{37}$ Die Siedler, die weggingen, zogen nach Buenos Aires, zum Teil in die Vereinigten Staaten oder nach Israel. Diese massive Abwanderung nach dem Krieg und Anfang der 50er Jahre ist nach Aussagen unserer Gesprächspartner auf verschiedene Gründe zurückzuführen: einerseits auf die Erschöpfung nach einem so schweren Existenzkampf, die fehlenden Perspektiven, die Isolierung, deren Mangel an Ausbildungsmöglichkeiten für die Kinder, die Absatzprobleme für die Produktion, gekoppelt mit schlechten Preisen für die Agrarprodukte. Auf der anderen Seite bot die Wiedergutmachung neue finanzielle Möglichkeiten. Durch diese massive Abwanderung der deutsch-jüdischen Emigranten verlor Avigdor seine Substanz. Die Kolonie besteht zwar heute noch, doch ist sie keine deutsch-jüdische Kolonie mehr.

Verglichen mit anderen südamerikanischen Ländern spielte Argentinien in der Kolonisation deutsch-jüdischer Emigranten eine einmalige Rolle: Auch wenn in Brasilien, Bolivien, Chile, Ecuador und in der Dominikanischen Republik andere landwirtschaftliche Projekte bestanden, so betrafen sie weit weniger jüdische Siedler als in Argentinien. Noch dazu scheiterten die meisten dieser Projekte, sieht man von Sosua in der Dominikanischen Republik und von der vornehmlich katholischen Siedlung Rolândia in Brasilien ab (Mühlen 1988, 104-109). Dagegen können die Kolonien deutsch-jüdischer Emigranten in Argentinien insgesamt als ein Erfolg betrachtet werden. Selbst wenn sie nur eine geringe Anzahl unter den deutsch-jüdischen Emigranten betrafen, die

\footnotetext{
${ }^{35}$ Interview Roberto Schopflocher.

${ }^{36}$ Alfred Neumeyer: Avigdor. In: Zehn Jahre Aufbauarbeit. 1943, 162.

${ }^{37}$ Interview Dr. Kurt Riegner, Buenos Aires, 9.1.1992.
} 
insgesamt in Argentinien Zuflucht fanden - und bei allen hervorgehobenen Mängeln haben diese landwirtschaftlichen Kolonien ihr wichtigstes Ziel erreicht, wie es ein Siedler aus Avigdor anläßlich des 50jährigen Bestehens der Kolonie im Semanario Israelita in Worte faßte:

Für uns jetzige und ehemalige Bewohner ist Avigdor das Symbol unserer Errettung, wo wir nach schwerer Not und Verfolgung ein neues Leben beginnen konnten, wo wir in einer Welt des Hasses und der Diskriminierung gut und freundlich aufgenommen wurden, um in Frieden und Freiheit eine neue Existenz aufzubauen, gleichberechtigt mit den restlichen Bewohnern des Landes. Und dafür sei immer wieder unser Dank zum Ausdruck gebracht. ${ }^{38}$

\section{Bibliographie}

Avni, Haim. 1983. Argentina y la historia de la inmigración judía (1810-1950). Buenos Aires: Universitaria Magnes.

[Cincuenta]. 1939. 50 años de colonización judía en la Argentina. Buenos Aires: DAIA.

Jewish Colonization Association (JCA). 1941. Jewish Colonization Association: Su obra en la república Argentina (1891-1941). Buenos Aires: Ayacucho.

Mühlen, Patrik von zur. 1988. Fluchtziel Lateinamerika. Die deutsche Emigration 1933-1945: politische Aktivitäten und soziokulturelle Integration. Bonn: Neue Gesellschaft.

Zehn Jahre Aufbauarbeit in Südamerika. 1943. Hg. anläßlich des zehnjährigen Bestehens der Asociación Filantrópica Israelita. Buenos Aires. 